\title{
Analysis of field to typical cable networks coupling in reverberation chamber's diffuse field
}

\author{
Xie xin \\ Research Institute of Electrostatic and Electromagnetic \\ Protection, Mechanical Engineering College, \\ Shijiazhuang, China \\ 332402911@qq.com \\ WANG Qing-guo \\ Research Institute of Electrostatic and Electromagnetic \\ Protection, Mechanical Engineering College, \\ Shijiazhuang, China \\ qwang_99@hotmail.com
}

\section{Jia Rui}

Research Institute of Electrostatic and Electromagnetic Protection, Mechanical Engineering College,

\section{Shijiazhuang, China}

piaoyang315@315.com

\begin{abstract}
For the study of the interference that complex electromagnetic environment coupling to multi-conductor transmission lines, further analysis of the laws of field coupling to lines. The reverberation chamber's chamber electromagnetic environment was simulated by scattering the incident angle of multiple incident waves. The Agrawal coupling theory was used to calculate the current response of the nodes of tree and annular networks. The reverberation chamber, spectrum analyzer, etc. were used to build the experimental system for verification. The result shows that: The "full-radiation" electromagnetic environment built by scattering the scattering the incident angle of multiple incident waves was similar to the field in reverberation chamber. When the transmitted signal is double exponential pulse, as the result of the mismatching loads, the time domain current response would be shock attenuation. The frequency where induced current peak appear had no significant relation with the pipe's length, the amplitude of the inductive current would attenuate with the increasing of frequency.
\end{abstract}

Keywords- reverberation chamber; cable networks; fullradiation; BLT equations; experiment

\section{INTRODUCTION}

The rapid development of electronic technology, the electromagnetic environment of human living space has become more and more complex. Communication building, life in the office building, business building, etc. are distributed transmission cable data communication, telephone, power line and so on. A lot of cables constitute a large distributed transmission network. In airplanes, automobiles, high-speed rail and aviation aircraft cockpit are also equipped with thousands of electronic devices and equipment. Most of these cable connect devices which are working under low voltage, high frequency, highly vulnerable to outside interference of electromagnetic radiation, lead to the equipment failure and even potential permanent damage (hard damage), the electromagnetic environment of the the sealed cavity with a lot of devices in is similar with the electromagnetic environmen in the reverberation chamber. The current domestic and foreign studies of reverberation chamber are mainly concentrated in the following aspects: On the one hand, the reverberation chamber electromagnetic environment modeling and simulation[1-3]. On the other hand reverberation indoor analysis and effect of electromagnetic field and cable coupling mechanism research. In reverberation chamber electromagnetic environment simulation, researchers at Research Institute of Electrostatic and Electromagnetic Protection used the electromagnetic simulation software FEKO to optimize the location of mechanical stirrer and antenna in the reverberation chamber[4]. Hua-bin Zhang from the sichuan university has used the monte carlo method[5] to simulate the electromagnetic environment of the reverberation chamber. Field coupling [6-8] problems basically has the following three research methods: One is half analytical and statistical methods; Secondly, the monte carlo method based on statistical principle; The third is based on the analytical method of electromagnetic reciprocity method. These three methods have large amount of calculation and complex formula, When comes to the research of cable network coupling rule, the calculation is bigger and the formula is more complicated. In this paper, by constructing multiincident plane waves to simulate the electromagnetic environment of reverberation chamber, The field coupling rule in the reverberation chamber was studied, which provide reference for cavity equipment protection.

\section{SIMULATION PRINCIPLE}

\section{A. The reverberation chamber electromagnetic environment simulation}

The reverberation chamber is an shielding cavity composed of high conductivity material, the traditional mechanical reverberation chamber is equipmented with the metal mixer. Electromagnetic wave spread in the reverberation chamber, reflect many times because of the inner wall of the cavity and metal mixer, in the work area of reverberation chamber, would have been radiated from all directions. As a new type of test site, the reverberation chamber is extensively applied in the electromagnetic compatibility test. The simulation of reverberation chamber[9-10] can not only convenient for experimental study, but also further for optimizing the design of the reverberation chamber, and provid guidance to enhance performance.

This article through to integral the incident Angle of wave spectrum of the testing area, by using the statistical characteristics of the angular spectrum to simulated the 
electromagnetic environment of the reverberation chamber.

$$
\stackrel{\mathbf{I}}{E}(\stackrel{\mathrm{r}}{r})=\int_{\Omega}^{\mathrm{uv}} F(\stackrel{\mathrm{v}}{k}) \exp (j k \stackrel{\mathrm{v}}{k} \cdot \stackrel{\mathrm{v}}{r}) d \Omega
$$

The spatial angle $4 \pi$ was devided into N parts, Each column of the space occupied by one incident angle, the electric field of the incident wave can be expressed by $\mathrm{N}$ columns' superimposition.

$$
\stackrel{\mathrm{r}}{E} \stackrel{\mathrm{r}}{r})=\sum_{n=1}^{N} \frac{4 \pi}{N} \stackrel{\mathrm{un}}{F}\left(\alpha_{n}, \beta_{n}\right) \exp \left[j \underset{k}{\mathrm{v}}\left(\alpha_{n}, \beta_{n}\right) \cdot \stackrel{\mathrm{v}}{r}\right]
$$

(2)is the discretization equation of (1), angle spectrum $F$ could be expressd:

$$
\stackrel{\text { uv }}{F}\left(\alpha_{n}, \beta_{n}\right)=\stackrel{\alpha}{\alpha} F_{\alpha}\left(\alpha_{n}, \beta_{n}\right)+\beta F_{\beta}\left(\alpha_{n}, \beta_{n}\right)
$$

$F_{\alpha}, \quad F_{\beta}$ denote the ponderance of $\stackrel{\text { uN }}{F}$ from $\stackrel{\text { wN }}{\alpha}, \stackrel{\text { wN }}{\beta}$ direction. Both of them fellow the $N\left(0, \sigma^{2}\right) \cdot \alpha, \beta$ fellow the evenly distributed in $[0,2 \pi]$. When the number of $\alpha, \beta$ taked under a sufficient number, the reverberation chamber's electromagnetic environment could be achieved. Fig .1 shows when there is 1000 stirring locations, each location has 1000 incident waves, the amplitude distribution of the electric field in three directions.

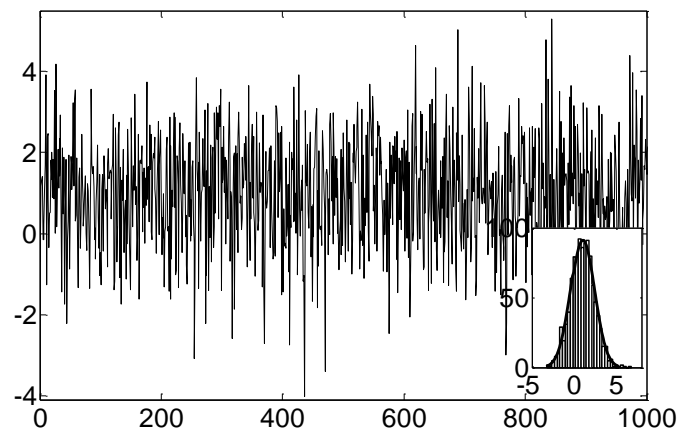

Figure 1. Electric fields simulated by Monte Carlo method and its distribution

\section{B. Muiltconductor transmission lines equation}

The frequency domain multi-conductor transmission lines equation [11]:

$$
\left\{\begin{array}{l}
\frac{d}{d z} V_{n}(z)=-Z_{n, m}^{\prime} I_{n}(z)+V_{n}^{(S)}(z) \\
\frac{d}{d z} I_{n}(z)=-Y_{n, m}^{\prime} V_{n}(z)+I_{n}^{(S)}(z) \circ
\end{array}\right.
$$

$V_{n}(z), I_{n}(z)$ denote voltage vector and current vector. $Z_{n, m}^{\prime}, Y_{n, m}^{\prime}$ denote the unit impedance matrix and unit admittance matrix of cable networks. $V_{n}^{(S)}(z)$, $I_{n}^{(S)}(z)$ denote the voltage source vector and current source vector.

General solution of ( 4 ) is as follows:

$$
\left\{\begin{array}{l}
\boldsymbol{V}=[\boldsymbol{I}+S][\Gamma-S]^{-1} \cdot V_{s} \\
I=Y_{c}[I-S][\Gamma-S]^{-1} \cdot V_{s}
\end{array}\right.
$$

$\boldsymbol{I}$ denotes unit matrix, $\boldsymbol{Y}_{\boldsymbol{c}}$ denotes characteristic admittance matrix, $\boldsymbol{\Gamma}$ denotes spread matrix, $\boldsymbol{V}_{\boldsymbol{s}}$ denotes Excitation matrix ; $\boldsymbol{S}$ denotes scattering matrix. The above parameters matrixs are determined by the structure of cable networks. The scattering matrix is the most difficult to solve. $\boldsymbol{S}_{t}$ and $\boldsymbol{S}_{\boldsymbol{a}}$ are the scattering matrix of the cable networks which were shown in Fig .2.

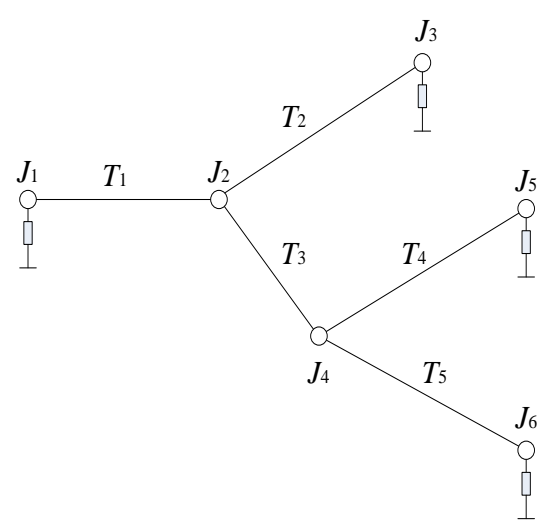

a) tree cable networks model

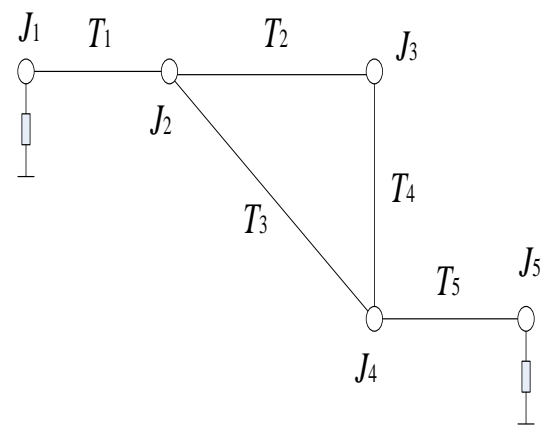

b) annular cable networks model

Figure 2. the model of typical-cable networks

$$
S_{t}=\left[\begin{array}{cccccccccc}
0 & s & 0 & 0 & 0 & 0 & 0 & 0 & 0 & 0 \\
-\frac{1}{3} & 0 & 0 & 0 & \frac{2}{3} & \frac{2}{3} & 0 & 0 & 0 & 0 \\
\frac{2}{3} & 0 & 0 & 0 & -\frac{1}{3} & \frac{2}{3} & 0 & 0 & 0 & 0 \\
\frac{2}{3} & 0 & 0 & 0 & \frac{2}{3} & -\frac{1}{3} & 0 & 0 & 0 & 0 \\
0 & 0 & s & 0 & 0 & 0 & 0 & 0 & 0 & 0 \\
0 & 0 & 0 & -\frac{1}{3} & 0 & 0 & 0 & 0 & \frac{2}{3} & \frac{2}{3} \\
0 & 0 & 0 & \frac{2}{3} & 0 & 0 & 0 & 0 & -\frac{1}{3} & \frac{2}{3} \\
0 & 0 & 0 & \frac{2}{3} & 0 & 0 & 0 & 0 & \frac{2}{3} & -\frac{1}{3} \\
0 & 0 & 0 & 0 & 0 & 0 & s & 0 & 0 & 0 \\
0 & 0 & 0 & 0 & 0 & 0 & 0 & s & 0 & 0
\end{array}\right]
$$




$$
S_{a}=\left[\begin{array}{cccccccccc}
0 & s & 0 & 0 & 0 & 0 & 0 & 0 & 0 & 0 \\
-\frac{1}{3} & 0 & 0 & 0 & \frac{2}{3} & \frac{2}{3} & 0 & 0 & 0 & 0 \\
\frac{2}{3} & 0 & 0 & 0 & -\frac{1}{3} & \frac{2}{3} & 0 & 0 & 0 & 0 \\
\frac{2}{3} & 0 & 0 & 0 & \frac{2}{3} & -\frac{1}{3} & 0 & 0 & 0 & 0 \\
0 & 0 & 0 & 0 & 0 & 0 & 0 & 1 & 0 & 0 \\
0 & 0 & 1 & 0 & 0 & 0 & 0 & 0 & 0 & 0 \\
0 & 0 & 0 & -\frac{1}{3} & 0 & \frac{2}{3} & 0 & 0 & 0 & \frac{2}{3} \\
0 & 0 & 0 & \frac{2}{3} & 0 & -\frac{1}{3} & 0 & 0 & 0 & \frac{2}{3} \\
0 & 0 & 0 & \frac{2}{3} & 0 & \frac{2}{3} & 0 & 0 & 0 & -\frac{1}{3} \\
0 & 0 & 0 & 0 & 0 & 0 & 0 & 0 & s & 0
\end{array}\right]
$$

Among them: $s=\frac{Z_{l}-Z_{c}}{Z_{l}+Z_{c}}$ 。

The Agrawal scartering formulas were used to calculate the source vector $S^{i}$ :

$S^{i}=-\frac{1}{2}\left(\frac{E_{0}(\cos \alpha \sin \varphi \cos \phi+\sin \alpha \sin \phi)^{j k d \sin \phi}}{\gamma-j k \cos \phi \cos \varphi}+E_{0} d \cos \varphi \cos \alpha\right)\left(1-e^{(\gamma-j k \cos \varphi \cos \phi) L}\right)$

(6)

\section{Simulation results}

Monte carlo statistical theory and Agrawal scattering formula were used to modeling the cable networks, the networks were composed of the copper wire whose radius was $0.5 \mathrm{~mm}$. The distance between ground and the cable networks was $0.8 \mathrm{~m}$. The length of each pipeline of the tree cable networks from T1 to T5 was 0.5 $\mathrm{m}, 0.8 \mathrm{~m}, 0.3 \mathrm{~m}, 0.6 \mathrm{~m}, 0.7 \mathrm{~m}$, the endpoint J1, J3, J5, J6 terminated $51 \Omega$ linear load. And the length of each pipeline of annular cable networks was $0.5 \mathrm{~m}, 0.8 \mathrm{~m}, 0.3$ $\mathrm{m}, 0.6 \mathrm{~m}, 0.7 \mathrm{~m}$. The endpoint $\mathrm{J} 1$ and $\mathrm{J} 5$ terminated $51 \Omega$ linear load. Fig .5 shows frequency domain current response waveform when there were 1000 stirrer position and in each stirrer position,there were 1000 incident planewave.

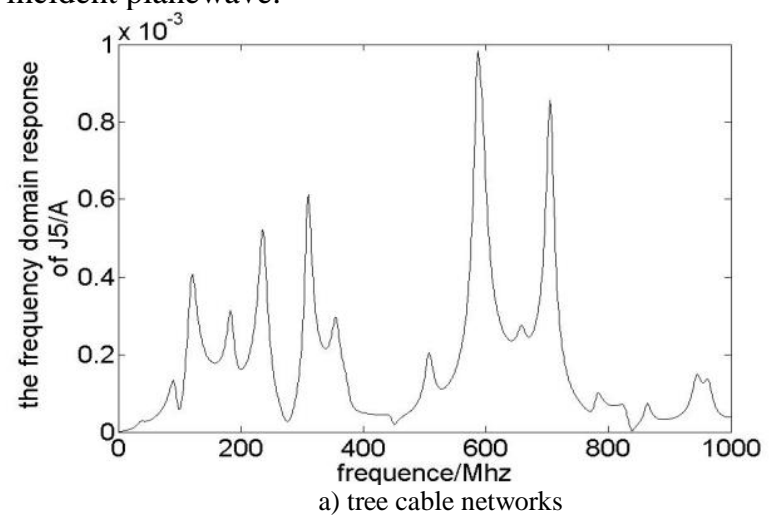

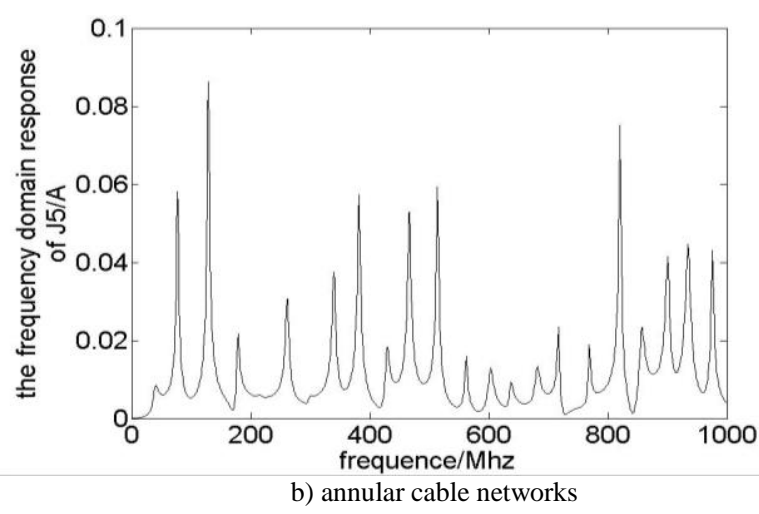

Figure 3. the frequency domain response of typical cable networks

So that the incident was a double exponential shape of unit amplitude polarized wave , the expression is:

$$
E^{i n c}(t)=1.05 \times\left(e^{-4 \times 10^{6} t}-e^{-4.76 \times 10^{8} t}\right)
$$

Its waveform is:

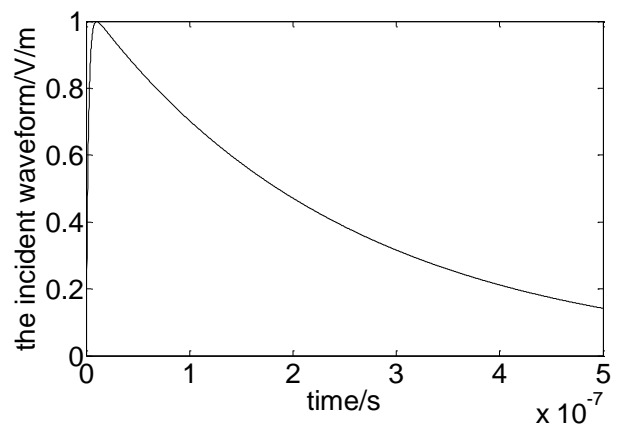

Figure 4. the incident waveform

Fourier transform was used to transform the time domain double index expression into the frequency domain. The expression in frequency domain multiplied the response of those networks. The current response spectrum was worked out. The time domain current response are obtained by Fourier inverse transformation. Fig .5 shows the time domain response of $\mathrm{J} 5$ of both tree and annular cable networks.

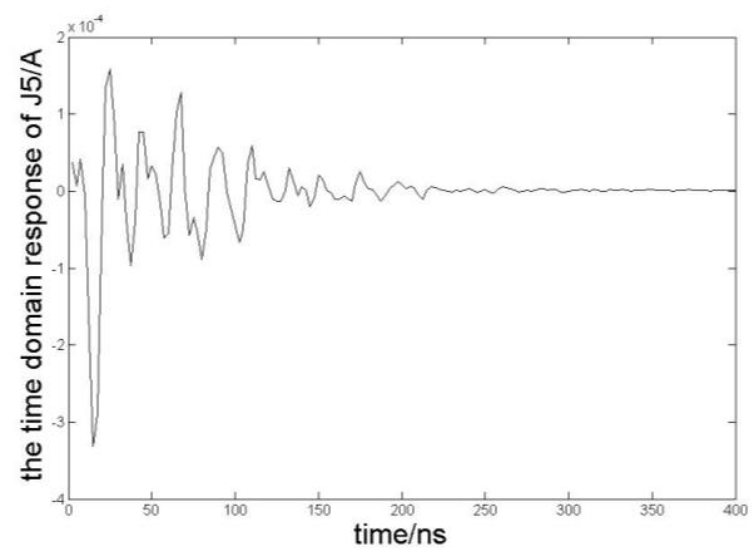

a) tree cable networks 


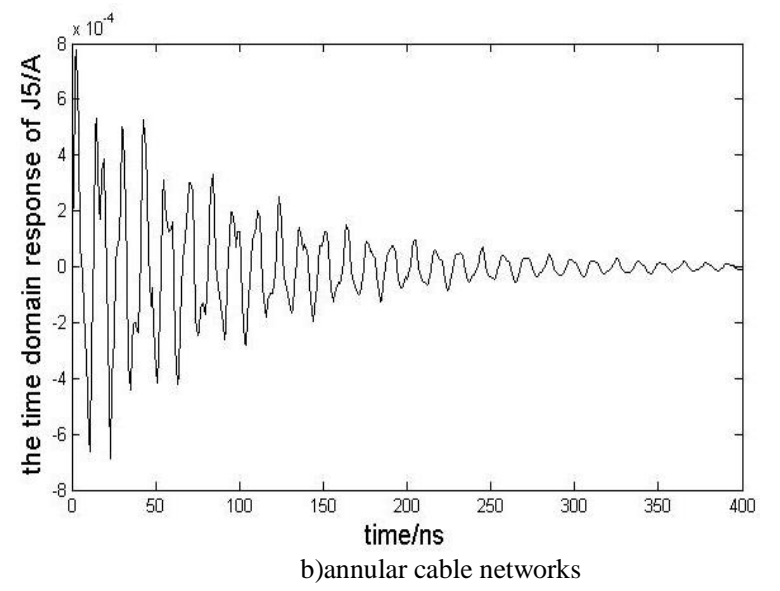

Figure 5. the time domain response

\section{EXPERIMENT}

The experiment used the size of $10.5 \mathrm{~m} * 8 \mathrm{~m} * 4.3$ $\mathrm{m}$ mechanical stirred rever-beration chamber. The main instruments includes: transmitting antenna,signal source, power amplifier. The current response signal acquisition instrument in-cludes: Tektronix model CT-1 type current probe (the testing frequency was $100 \mathrm{kHz}-1 \mathrm{GHz}$ ), photoelectric converter, electro-optical converter, optical fiber, ground floor, oscilloscope. The measured tree type cable network parameters and the simulation was consistent, in order to obtain exacter current sensing signal of each node cable network, decrease the experimental error caused by the current probe inducting signal, the current probe was placed in a shield box, Fig .6 C) gave the circuit inside the shield box.
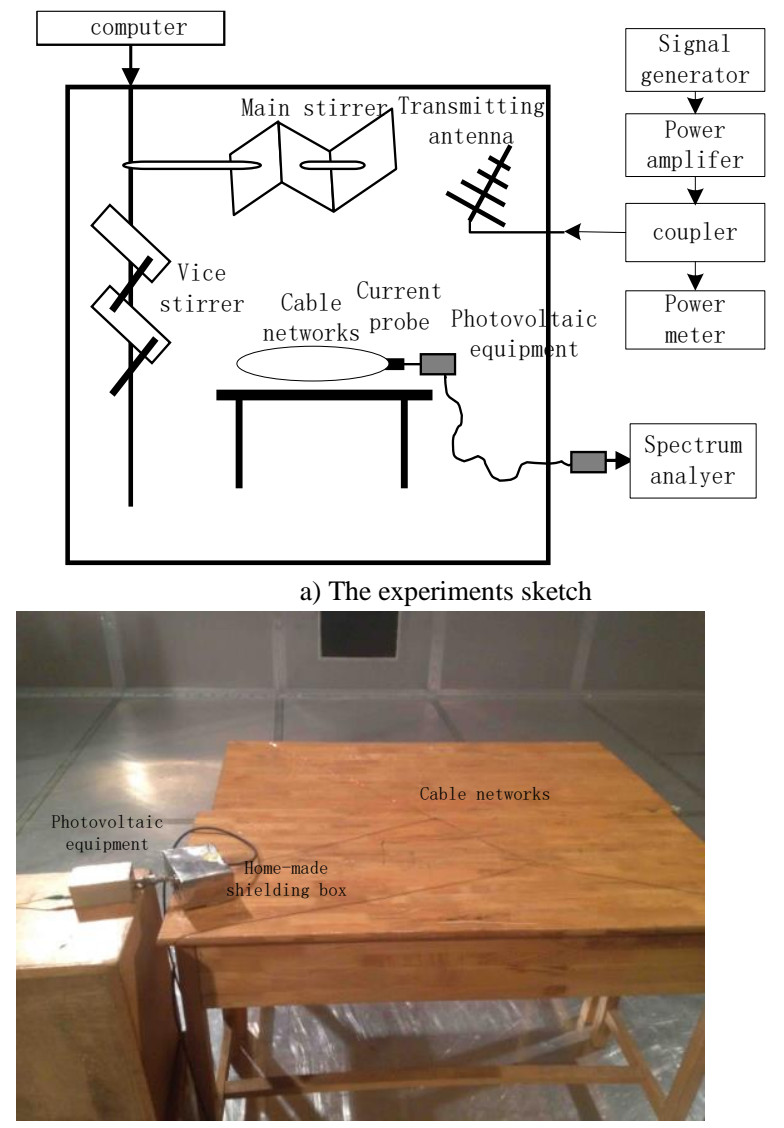

b) experiment environment

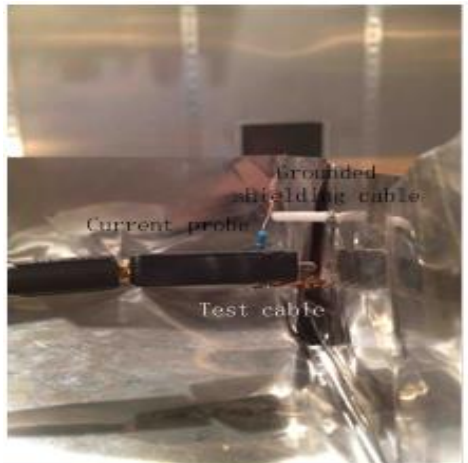

c) the sketch of inner-shielding box

Figure 6. The experiments sketch of the coupling of field to line in reverberation chamber

\section{VERIFICATION AND COMPARISON}

In order to verify the reliability of the simulation method, further analysis the rules of the cable networks' responses in the reverbration chamber. The test frequency band was $100 \mathrm{Mhz}-1 \mathrm{Ghz}$, took the $5 \mathrm{Mhz}$ as the frequency step.The stirrer worked in step mode. The main mixer seted three mixing location,every location there were four mixing location for vice stirrer. The current response and input power of each mixing location were noted. The input power was used to normalize the current response with (8).

$$
I^{\prime}=\frac{I}{\sqrt{P}}
$$

Fig .7 shows the frequency domain node 5 normalized current response curve of tree cable network.

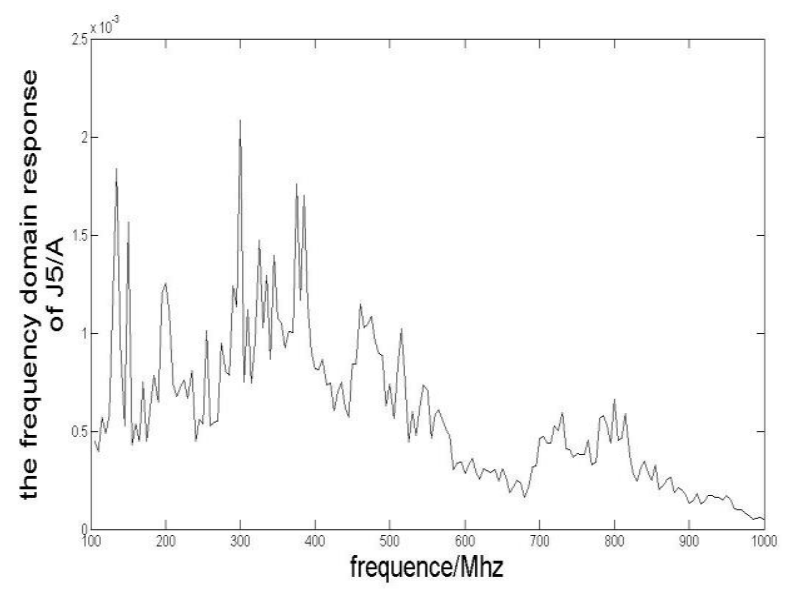

Figure 7. the real response of J5 of tree cable networks

\section{SUMMARY}

The theory that discreting the space angle of incident waves was used to simulate the electromagnetic environment in the reverberation chamber, the Agrawal scattering formula was used to caculate the transmission lines equation. The method that using spectrum analyzer, power meter, the photoelectric conversion device to test the coupling signal of cable networks was puted forward. Compare the simulation and experimental results, the following conclusions could be gotten. The simulation methods were reliable. When the incident signal was double exp-onential pulse, the time domain current 
response would be shock attenuation due to mismatch of the load mismatch. The frequency point current peak appears did not have significant relationship with the length of the pipe. With increasing of the frequence, the current sense signal would be attenuation. Because of the crude experimental devices, there were differences between the simulation resu-lts and the experimental results, this was what should be improve next. The model in this paper was useful for the conductor in homogeneous medium rather than in the inhomogeneous medium[12]. As a result, the inhomogeneous medium conditions remains to be further research.

\section{REFERENCES}

[1] PARMANTIER J-P. Numerical coupling models for complex systems and results [J]. IEEETransElectromagnCompat, Aug.2004, 46(3): 359-367.

[2] Zhang Wei,Du Zheng-Wei.Simulation of irradiation effects of high power microwave on PCB circuits. High power laser and particle beams. 2011,(11): 2841-2844.

[3] LIANG Shuang-Gang,XU Jia-Dong,NIU Yi-Yong,et al.Simulation analysis and experimental investigation of SSRC[J].Chinese journal of radio science. 2010,(06): 1058-1063.

[4] CUI Yao-Zhong,WEI Guang-Hui,FAN Li-Si, et al..Effect of Field Performance in Reverbration Chamber Excited by Two Transmitting Antennas.Journal of Microwaves. 2011, (02): 2934.
[5] ZHANG Hua-bin, ZHAO Xiang, ZHOU Hai-jing, et al. . Probabilistic and statistical analysis of mode stirred reverberation chamber and its Monte Carlo simulation[J]. High power laser and particle beams, 2011, 23 (9) : 2475-2480.

[6] WU Zhen-Jun, WANG Li-Fang, Liao Cheng-Lin.A nowel FDTD method for multi-conductor transmission lines terminating in frequency-dependent loads[J].Acta Physica Sinica. 2009, (09): 6146-6151.

[7] LUO Qing-chun,ZHAO Xiang,YAN Li-Ping, et al.ProbabilisticStatistical model of reverberation chamber based on cavity mode theory and the monte carlo simulation[J].Journal of Sichuan University(Natural Science Edition). 2013,(04): 770-774.

[8] WENG Ling-Wen,NIU Zhong-xia,ZHOU Dong-Fang,LIN Jing$\mathrm{Yu}$. et al.Analysis of the Electromagnetic Coupling in Transmission Line Networks[J]. 2005, (17): 66-67.

[9] WAN Bo,BAI Rong-Feng,ZHANG Long, et al.Simulation and Experimental Verification of Reverberation Chamber[J].Journal of CAEIT.2013, 01): 106-110.

[10] LIN Gao-Sheng,QIN Yu-Jian,JIANG Yao-Ming, et al.Method of BLT Equation for Solving Responses of Transmission Networks[J].Space electronic technology. 2012,(04): 62-66.

[11] FREDERICK M.TESCHE M V L, TORBJORN KARLSSON. EMC Analysis Methods and Computational Models [M].

[12] ZHENG Qin-Hong,ZENG Hua,JIANG Shao-Qian, et al.Capacitance calulation for transmission line filled with piecewise homogeneous dielectric using boundary element method.High power laser and particle beams[J], 2005, (06):909912 